UDC 517.51

E. NEUMAN

\title{
WILKER AND HUYGENS-TYPE INEQUALITIES INVOLVING GUDERMANNIAN AND THE INVERSE GUDERMANNIAN FUNCTIONS
}

\begin{abstract}
Five Wilker Huygens-type inequalities involving Gudermannian and the inverse Gudermannian functions are obtained. The Schwab-Borchardt mean plays a crucial role in the proofs. Also, an analytical inequality for the sums of powers, established earlier by this author, is an indispensable tool in the the proofs of the main results of this paper.
\end{abstract}

Key words: Gudermannian function, inverse Gudermannian function, Wilker inequality, Schwab-Borchardt mean, Huygens inequality, inequalities

2010 Mathematical Subject Classification: 26D07, 26E60

1. Introduction. In recent years a significant progress has been made in the area of inequalities involving circular and hyperbolic functions. In particular, the following results

$$
2<\left(\frac{\sin x}{x}\right)^{2}+\frac{\tan x}{x}
$$

and

$$
3<2 \frac{\sin x}{x}+\frac{\tan x}{x}
$$

$\left(0<|x|<\frac{\pi}{2}\right)$ have attracted attention of several researchers. Inequalities (1) and (2) have been obtained by J. B. Wilker [23] and C. Huygens [5], respectively. Several proofs of these results can be found in mathematical literature (see, e.g., [4, 8, 9, 17, 20, 22, 24, 25, 31, 32, 33, 30, 34, 35, 36. and the references therein). 
Counterparts of (1) and (2) for the hyperbolic functions have also been obtained. They read as follows

$$
2<\left(\frac{\sinh x}{x}\right)^{2}+\frac{\tanh x}{x}
$$

and

$$
3<2 \frac{\sinh x}{x}+\frac{\tanh x}{x}
$$

$(x \neq 0)$. For the proofs of these results the interested reader is referred to [35] and [17, respectively.

Generalizations of inequalities (1) - (4) are known. See [8, 9, 26, 27, 28, 29] and the references therein. Wilker and Huygens- type inequalities have also been established for the lemniscate functions, generalized Jacobian elliptic, generalized trigonometric and generalized hyperbolic functions and also for the theta functions. The interested reader is referred to [10, 11, 12, 13, 14, 18.

The goal of this paper is to obtain some inequalities which bear resemblance of inequalities (1) - (4) for the Gudermannian and the inverse Gudermannian functions. Definitions of these functions are given in Section 2. Also, some preliminary results are included in this section. The Wilker-Huygens inequalities involving functions under discussion are obtained in Section 4.

2. Definitions and preliminaries. We begin this section with definitions of the Gudermann function and its inverse function. Throughout the sequel they will be denoted by $g d$ and $g d^{-1}$, respectively. Following [19, 4.23 (viii)]

$$
g d(x)=\int_{0}^{x} \frac{1}{\cosh t} d t, \quad x \in \mathbb{R}
$$

and

$$
g d^{-1}(x)=\int_{0}^{x} \frac{1}{\cos t} d t, \quad|x|<\pi / 2 .
$$

It is known (see, e.g., [19, 4.23 (viii)]) that

$$
g d(x)=\sin ^{-1}(\tanh x)=\tan ^{-1}(\sinh x)=2 \tan ^{-1}\left(\tanh \frac{1}{2} x\right)
$$


and

$$
g d^{-1}(x)=\sinh ^{-1}(\tan x)=\tanh ^{-1}(\sin x) .
$$

In the proofs presented in this section we will utilize the following four theorems. The first result reads as follows [18, 21]:

Theorem 1. Let $f$ be a positive and strictly monotonic function defined on the subset $I$ of the positive semiaxis. Assume that $f^{-1}$ is strictly increasing and also that $f(x) \geq x(f(x) \leq x)$ for all $x \in I$. If the function $g(x)=f(x) / x$ is increasing (decreasing) on $I$, then

$$
\frac{f(x)}{x} \geq \frac{x}{f^{-1}(x)}
$$

Inequality (9) is reversed if the function $g(x)$ is decreasing (increasing) on $I$.

We shall employ the following theorem (see [1]):

Theorem 2. Let the functions $f$ and $g$ be continuous on $[c, d]$, differentiable on $(c, d)$ and such that $g^{\prime}(t) \neq 0$ on $(c, d)$. If $\frac{f^{\prime}(t)}{g^{\prime}(t)}$ is (strictly) increasing (decreasing) on $(c, d)$, then the functions $\frac{f(t)-f(d)}{g(t)-g(d)}$ and $\frac{f(t)-f(c)}{g(t)-g(c)}$ are also (strictly) increasing (decreasing) on $(c, d)$.

Theorem 3. Let $u, v, \alpha, \beta, \gamma$ and $\delta$ be positive numbers which satisfy the following conditions

(i) $\min (u, v)<1<\max (u, v)$,

(ii) $1<u^{\gamma} v^{\delta}$.

Then the inequality

$$
\alpha+\beta<\alpha u^{p}+\beta v^{q}
$$

is satisfied provided $u<1<v$ and

$$
q>0 \quad \text { and } \quad p \alpha \delta \leq q \beta \gamma .
$$

If in addition the positive numbers $u, v, \gamma$ and $\delta$ are such that (iii) $\gamma+\delta<\gamma \frac{1}{u}+\delta \frac{1}{v}$

then the inequality (10) is satisfied if

$$
p \leq q \leq-1 \quad \text { and } \quad \beta \gamma \leq \alpha \delta
$$

(see [9], Theorem 3.1). 
Conditions of validity of $(10)$ when $v<1<u$ are also obtained in [9]. The counterpart of (11) is

$$
p>0 \quad \text { and } \quad q \beta \gamma \leq p \alpha \delta
$$

while the conditions (12) are replaced now by

$$
q \leq p \leq-1 \quad \text { and } \quad \alpha \delta \leq \beta \gamma .
$$

Also, we shall employ the following result [9]:

Theorem 4. Let $u, v, \gamma$ and $\delta$ be positive numbers which satisfy conditions (i)-(iii) of Theorem 3. Then the inequality

$$
2<\left(\frac{1}{u}\right)^{\gamma p}+\left(\frac{1}{v}\right)^{\delta p}<u^{\gamma p}+v^{\delta p}
$$

holds true provided $\gamma \geq 1, \delta \geq 1$, and $p \geq 1$. Second inequality in (15) is valid if $p>0$.

3. Schwab-Borchardt mean. In what follows the letters $a$ and $b$ will always stand for positive and unequal numbers.

The important mean utilized in this paper is called the Schwab-Borchardt mean and is defined as follows:

$$
S B(a, b) \equiv S B= \begin{cases}\frac{\sqrt{b^{2}-a^{2}}}{\cos ^{-1}(a / b)} & \text { if } a<b, \\ \frac{\sqrt{a^{2}-b^{2}}}{\cosh -1(a / b)} & \text { if } b<a\end{cases}
$$

(see, e.g., 2], 3]). It is well known that the mean $S B$ is strict, nonsymmetric and homogeneous of degree one in its variables.

For the reader's convenience we will record now some inequalities satisfied by the Schwab-Borchardt mean. They are obtained in [15, 16]. See also [13. They read as follows

$$
\begin{gathered}
\left(a b^{2}\right)^{1 / 3}<(b S B(b, a))^{1 / 2}<S B(a, b), \\
3(b+S B(b, a))<2(a+2 b),
\end{gathered}
$$

and

$$
S B(a, b)<S B(b, a)
$$


provided $a>b$. If the last inequality is satisfied then more inequalities involving mean $S B$ is known. We record below one of them

$$
2 S B(a, b)<b+S B(b, a)
$$

(see, e.g., [7]).

4. Main Results. Our first result reads as follows:

Theorem 5. Let $\alpha, \beta>0$. Then the inequality

$$
\alpha+\beta<\alpha\left(\frac{g d(x)}{x}\right)^{p}+\beta\left(\frac{g d^{-1}(x)}{x}\right)^{q} \quad(0<|x|<\pi / 2)
$$

holds true provided

$$
q>0 \quad \text { and } \quad p \alpha \leq q \beta .
$$

Proof. We shall establish the assertion using Theorem 3 with

$$
u=\frac{g d(x)}{x} \quad \text { and } \quad v=\frac{g d^{-1}(x)}{x} .
$$

Firstly we shall prove that $u$ and $v$ satisfy the separation condition

$$
u<1<v
$$

For the proof of the left inequality in (24) we introduce function $h(x)=$ $=x-g d(x)$. Differentiation, with use of (5), yields

$$
h^{\prime}(x)=1-\frac{1}{\cosh x} .
$$

Clearly $h^{\prime}(x)>0$ for all $0<|x|<\pi / 2$. This in conjunction with $h(0)=0$ implies that $h(x) / x>0$ or what is the same that $u<1$. The second inequality in (24) can be established in a similar way using the function $h(x)=g d^{-1}(x)-x$. We omit further details. We shall prove now that $u$ and $v$ satisfy condition (ii) of Theorem 3 with $\gamma=\delta=1$. To this aim we will utilize Theorem A with $f(x)=g d(x)$. Clearly function $f^{-1}$ is strictly increasing on its domain. Also, the inequality $u<1$ implies $f(x)<x$. In order to apply inequality (9) we have to prove that the function

$$
\frac{f(x)}{x}=\frac{g d(x)}{x}
$$


is decreasing. Using Theorem 2 we get easily

$$
\frac{g d^{\prime}(x)}{x^{\prime}}=\frac{1}{\cosh (x)} .
$$

This implies that $f(x) / x$ is a decreasing function. With the notation introduced above we see that the inequality (9) can be written as

$$
1<u v \text {. }
$$

This shows that the condition (ii) of Theorem 3 is satisfied provided $\gamma=$ $=\delta=1$. Conditions (22) of validity Theorem 5 follow immediately from (11). The proof is complete.

We shall now prove the next result.

Theorem 6. The inequality

$$
\alpha+\beta<\alpha\left(\frac{\sin x}{g d^{-1}(x)}\right)^{p}+\beta\left(\frac{\tan x}{g d^{-1}(x)}\right)^{q} \quad(|x|<\pi / 2)
$$

is valid if either the numbers $p, q$ and the positive numbers $\alpha$ and $\beta$ satisfy the conditions

$$
q>0 \quad \text { and } \quad 2 p \alpha \leq q \beta
$$

or if

$$
p \leq q \leq-1 \quad \text { and } \quad \beta \leq 2 \alpha .
$$

Proof. Let the numbers $a$ and $b$ be such that $a<b$. This yields

$$
a<S B(b, a)<b .
$$

Letting

$$
u=\frac{S B(b, a)}{b} \quad \text { and } \quad v=\frac{S B(b, a)}{a} .
$$

This yields

$$
u<1<v .
$$

With $u$ and $v$ as defined in (28) we see that the inequality (17) implies

$$
1<u v^{2} .
$$

Thus $u$ and $v$ satisfy condition (ii) of Theorem 3 with

$$
\gamma=1 \quad \text { and } \quad \delta=2 .
$$


Finally with $u$ and $v$ as defined above we see that the condition (18) can be written as follows

$$
3<\frac{1}{u}+2 \frac{1}{v} .
$$

To complete the proof we let $a=\cos x$ and $b=1$. Utilizing the formula

$$
S B(1, \cos x)=\frac{\sin x}{g d^{-1}(x)}
$$

(see [6]) and applying Theorem 3 we obtain the asserted result. This completes the proof.

Our next result reads as follows:

Theorem 7. The inequality

$$
\alpha+\beta<\alpha\left(\frac{\sinh x}{g d(x)}\right)^{p}+\beta\left(\frac{\tanh x}{g d(x)}\right)^{q} \quad(x \in \mathbb{R})
$$

is valid if either the numbers $p, q$ and the positive numbers $\alpha$ and $\beta$ satisfy the conditions

$$
p>0 \quad \text { and } \quad q \beta \leq 2 p \alpha
$$

or if

$$
q \leq p \leq-1 \quad \text { and } \quad 2 \alpha \leq \beta .
$$

Proof. Assume that the positive numbers $a$ and $b$ are such that $b<a$. This implies

$$
b<S B(b, a)<a .
$$

With $u$ and $v$ as defined in (28) we see that now

$$
v<1<u \text {. }
$$

To verify that $u$ and $v$ satisfy conditions (ii) and (iii), with

$$
\gamma=1 \quad \text { and } \quad \delta=2 \text {, }
$$

of Theorem 3 we proceed exactly in the same way as in the proof of Theorem 6 . To complete the proof we let $a=\cosh x(x \in \mathbb{R})$ and $b=1$. Making use of the formula

$$
S B(1, \cosh x)=\frac{\sinh x}{g d(x)}
$$


(see [6]) we obtain easily the assertion using inequalities (13) and (14) of Theorem 6 .

We shall now prove the following result.

Theorem 8. The inequality

$$
\alpha+\beta<\alpha\left(\frac{x}{g d^{-1}(x)}\right)^{p}+\beta\left(\frac{\tan x}{g d^{-1}(x)}\right)^{q} \quad(|x|<\pi / 2)
$$

is valid if either the numbers $p, q$ and the positive numbers $\alpha$ and $\beta$ satisfy the conditions

$$
q>0 \quad \text { and } \quad p \alpha \leq q \beta
$$

or if

$$
p \leq q \leq-1 \quad \text { and } \quad \beta \leq \alpha .
$$

Proof. Let $a>b>0$. Then (19) yields

$$
b<S B(a, b)<S B(b, a) .
$$

We define now

$$
u=\frac{S B(a, b)}{S B(b, a)} \quad \text { and } \quad v=\frac{S B(a, b)}{b} .
$$

Clearly

$$
u<1<v \text {. }
$$

Using the second inequality in (17) we get easily

$$
1<u v \text {. }
$$

Thus the condition (ii) of Theorem 3 is satisfied with

$$
\gamma=\delta=1
$$

Inequality (20) together with the definitions of $u$ and $v$ yield

$$
2<\frac{1}{u}+\frac{1}{v}
$$

We now let $a=1 / \cos x(|x|<\pi / 2)$ and $b=1$. Taking into account that

$$
S B\left(\frac{1}{\cos x}, 1\right)=\frac{\tan x}{g d^{-1}(x)}
$$


(see [6]) we obtain the asserted result employing Theorem $\mathrm{C}$ again.

We close this section with the following:

Theorem 9. The two-sided inequality (15) holds true if $\gamma=\delta=1$ and if either

$$
(u, v)=\left(\frac{g d(x)}{x}, \frac{g d^{-1}(x)}{x}\right)
$$

or if

$$
(u, v)=\left(\frac{x}{g d^{-1}(x)}, \frac{\tan x}{g d^{-1}(x)}\right) .
$$

Also, the two-sided inequality (15) holds true if $\gamma=1, \delta=2$ and if either

$$
(u, v)=\left(\frac{\sin x}{g d^{-1}(x)}, \frac{\tan x}{g d^{-1}(x)}\right)
$$

or if

$$
(u, v)=\left(\frac{\sinh x}{g d(x)}, \frac{\tanh x}{g d(x)}\right) .
$$

Proof. Apply Theorem 4 to Theorems 5, 8, 6, and 7, respectively. We omit further details.

The Wilker Huygens-type inequalities for the following pairs of functions

$$
(u, v)=\left(\frac{g d(x)}{x}, \frac{\sinh x}{x}\right)
$$

and

$$
(u, v)=\left(\frac{g d^{-1}(x)}{x}, \frac{\sin x}{x}\right) .
$$

are established in [6] by means different than those used in this paper.

Acknowledgment. This author is deeply indebted to an anonymous referee for constructive remarks which have been used to improve a first version of this work.

\section{References}

[1] Anderson G. D., Vamanamurthy M. K., Vuorinen M. Monotonicity rules in calculus. Amer. Math. Monthly, 2006, vol. 133, no. 9, pp. 805-816.

[2] Borwein J. M., Borwein P. B. Pi and the AGM: A Study in Analytic Number Theory and Computational Complexity. John Wiley and Sons, 1987. 
[3] Carlson B. C. Algorithms involving arithmetic and geometric means. Amer. Math. Monthly, 1971, vol. 78, no. 5, pp. 496-505. DOI: 10.2307/2317754.

[4] Guo B.-N., Qiao B.-M., Qi F., and Li W. On new proofs of Wilker inequalities involving trigonometric functions. Math. Inequal. Appl., 2003, vol. 6, no. 1 , pp. 19-22.

[5] Huygens C. Oeuvres Completes 1888-1940, Société Hollondaise des Science, Haga.

[6] Neuman E. Inequalities for the Schwab-Borchardt mean and their applications. J. Math. Inequal., 2011, vol. 5, no. 4, pp. 601-609.

[7] Neuman E. Refinements and generalizations of certain inequalities involving trigonometric and hyperbolic functions. Adv. Inequal. Appl., 2012, vol. 1, no. 1, pp. 1-11.

[8] Neuman E. On Wilker and Huygens type inequalities. Math. Inequal. Appl., 2012, vol. 15, no. 2, pp. 271-279.

[9] Neuman E. Inequalities for weighted sums of powers and their applications. Math. Inequal. Appl., 2012, vol. 24, no. 4, pp. 995-1005.

[10] Neuman E. On lemniscate functions. Integral Transforms Spec. Funct., 2013, vol. 15, no. 3, pp. 164-171.

[11] Neuman E. Wilker and Huygens type inequalities for Jacobian elliptic and theta functions. Integral Transforms Spec. Funct., 2014, vol. 25, no. 3, pp. 240-248.

[12] Neuman E. Inequalities involving generalized Jacobian elliptic functions. Integral Transforms Spec. Funct., 2014, vol. 25, no. 11, pp. 864-873.

[13] Neuman E. Wilker and Huygens type inequalities for the generalized trigonometric and for the generalized hyperbolic functions. Appl. Math. Comput., 2014, vol. 230, pp. 211-217.

[14] Neuman E. Wilker and Huygens type inequalities for some elementary functions and Eulerian numbers. Adv. Studies Contemp. Math., 2015, vol. 25, no. 2, pp. 189-194.

[15] Neuman E., Sándor J. On the Schwab-Borchardt mean. Math. Pannon., 2003, vol. 14, no. 2, pp. 253-266.

[16] Neuman E., Sándor J. On the Schwab-Borchardt mean II. Math. Pannon., 2006, vol. 17, no. 1, pp. 49-59.

[17] Neuman E., Sándor J. On some inequalities involving trigonometric and hyperbolic functions with emphasis on the Cusa-Huygens, Wilker and Huygens inequalities. Math. Inequal. Appl., 2010, vol. 13, no. 4, pp. 715-723.

[18] Neuman E., Sándor J. Inequalities involving Jacobian elliptic functions and their inverses. Integral Transforms Spec. Functs., 2012, vol. 23, no. 10, pp. 719-722. 
[19] Olver F. W. J., Lozier D. W., Boisvert R. F., Clark C. W., editors. NIST handbook of mathematical functions. Cambridge University Press, 2010.

[20] Pinelis I. L'Hôpital rules of monotonicity and Wilker-Anglesio inequality. Amer. Math. Monthly, 2004, vol. 111, pp. 905-909.

[21] Sándor J. private communication.

[22] Sumner J. S., Jagers A. A., Vowe M., and Anglesio J. Inequalities involving trigonometric functions. Amer. Math. Monthly, 1991, vol. 98, pp. 264-267.

[23] Wilker J. B. Problem E 3306. Amer. Math. Monthly, 1989, vol. 96, article 55 .

[24] Wu S. On extension and refinement of Wilker inequality. Rocky Mountain Math. J., 2009, vol. 39, no. 2, pp. 683-687.

[25] Wu S., Baricz A. Generlizations of Mitrinović, Adamović and Lazarevic's inequalities and their applications. Publ. Math. Debrecen, 2009, vol. 75, no. 3-4, pp. 447-458.

[26] Wu S.-H., Debnath L. A new generalized and sharp version of Jordan's inequality and its applications to the improvement of the Yang Le inequality. Appl. Math. Lett., 2006, vol. 19, no. 12, pp. 1378-1384.

[27] Wu S.-H., Debnath L. A new generalized and sharp version of Jordan's inequality and its applications to the improvement of the Yang Le inequality II. Appl. Math. Lett., 2007, vol. 20, no. 5, pp. 532-538.

[28] Wu S.-H., Debnath L. Jordan-type inequalities for differentiable functions and their applications. Appl. Math. Lett., 2008, vol. 21, no. 8, pp. 803-809.

[29] Wu S.-H., Debnath L. Wilker-type inequalities for hyperbolic functions. Appl. Math. Lett., 2012, vol. 25, no. 5, pp. 837-842.

[30] Wu S.-H. and Srivastava H. M. A weighted and exponential generalization of Wilker's inequality and its applications. Integral Transform. and Spec. Funct., 2007, vol. 18, no. 8, pp. 525-535.

[31] Wu S.-H., Srivastava H. M. A further refinement of Wilker's inequality. Integral Transforms and Spec. Funct., 2008, vol. 19, no. 10, pp. 757-765.

[32] Wu S.-H., Srivastava H. M. A further refinement of a Jordan type inequality and its application. Appl. Math. Comput., 2008, vol. 197, no. 2, pp. 914923.

[33] Wu S.-H., Srivastava H. M., Debnath L. Some refined families of Jordantype inequalities and their applications. Integral Transforms Spec. Funct., 2008, vol. 19, no. 3, pp. 183-193.

[34] Zhu L. A new simple proof of Wilker's inequality. Math. Inequal. Appl., 2005, vol. 8, no. 4, pp. 749-750. 
[35] Zhu L. On Wilker-type inequalities. Math. Inequal. Appl., 2007, vol. 10, no. 4, pp. $727-731$.

[36] Zhu L. Some new Wilker type inequalities for circular and hyperbolic functions. Abstract Appl. Analysis, 2009, vol. 2009, 9 p. DOI: $10.1155 / 2009 / 485842$.

Received April 11, 2017.

In revised form, June 6, $201 \%$.

Accepted June 6, $201 \%$.

Published online June 20, $201 \%$.

Mathematical Research Institute

144 Hawthorn Hollow, Carbondale IL 62903, USA

E-mail: edneuman76@gmail.com 